\title{
Development Of Novice Teachers' Views Of Students Ideas As Sensible And Productive
}

\author{
Clarissa E. Lovegren and Amy D. Robertson
}

Seattle Pacific University Department of Physics, 3307 Third Ave W, Seattle, WA 98119-1997

\begin{abstract}
Novice teachers in Seattle Pacific University's (SPU's) Learning Assistant (LA) Program shifted their views of student thinking over the course of two academic quarters. LAs originally valued student ideas as (1) a part of caring for students as whole people and (2) instrumental for diagnosing misunderstandings. As the second quarter of the course proceeded, LAs highlighted the intellectual value of student thinking, treating student ideas as sensible and productive. This paper proposes plausible mechanisms that foster this expanded view. In particular, we suggest that articulating teaching values that prioritize student reasoning, searching for "kernels of correctness" in student thinking, and negotiating curricular knowledge promote this particular attention towards student ideas.
\end{abstract}

Keywords: learning assistants, novice teachers, teaching values, teacher attention, student ideas, curricular knowledge PACS: 01.40.Fk, 01.40.Ha, 01.40.jc

\section{INTRODUCTION}

Over the course of two academic quarters, Seattle Pacific University (SPU) Learning Assistants (LAs) expanded their views of student thinking. Initially, LAs valued student ideas as (1) a part of caring for students as whole people and (2) instrumental for diagnosing misunderstandings. LAs shifted their perceptions to include intellectually valuing student ideas. When LAs treat student ideas as sensible and as productive starting places for instruction, they are intellectually valuing those ideas.

Teaching that is grounded in a view of student ideas as intellectually valuable has a number of desirable consequences. The student is treated as intelligent and capable which aligns with theories of learning, e.g., constructivism [1]. This mindset towards teaching also promotes conceptual learning and positive attitudes toward the discipline [2,3].

The goal of this paper is to propose and illustrate mechanisms that successfully fostered a view of student thinking as intellectually valuable among novice teachers (LAs) at SPU. We use excerpts from LAs' teaching reflections to identify three specific mechanisms: the articulation of teaching values that prioritize student reasoning, the search for kernels of correctness in student thinking, and the intentional development of curricular knowledge.

Although our research community aims to promote views of student thinking as productive and resourceful, little research has been conducted to understand how these views might be fostered. We begin such work in this paper with an in-depth, descriptive study of one context in which these views were promoted. These mechanisms could inform teacher preparation efforts at other institutions that have similar goals.

The three mechanisms were embedded within the specific context of the SPU Physics LA Program. We suspect that the enactment and details of the mechanisms were influenced by the program's culture. Thus, before we articulate the mechanisms, we describe the SPU LA Program philosophy and culture.

\section{PHILOSOPHY AND CULTURE OF SPU LA PROGRAM}

The mechanisms that fostered the shift in LAs' views of student ideas were rooted in the culture of the SPU LA Program. This selective program places student leaders in SPU's introductory physics courses as instructional assistants. Currently, about twenty LAs are recruited each year. SPU LAs are typically undergraduate STEM majors who have passed the equivalent courses in which they are placed. The program is modeled after the LA Program at CUBoulder [4] and contains three primary components. Content preparation is a weekly planning course with physics instructors. Pedagogy is a weekly course in science education theory and its application. Practice provides time for LAs to work with students through in-class instruction and tutoring.

The SPU LA pedagogy and content prep courses are approached from a theoretical framework that blends responsive teaching, an ethic of care, and a view of learning as participation. The program instructor models responsive teaching by seeking to understand the meaning LAs are making of their teaching experiences. During class-wide discussions, 
the instructor connects LAs' ideas to one another and to the discipline of Physics Education Research $[5,6]$. The direction that the class takes in the short- and long-term is determined by LAs' developing ideas $[7,8]$.

Responsive teaching at SPU is embedded in a general ethic of care between the course instructor and LAs, and between LAs themselves [9,10]. In particular, LAs seek to understand one another's ideas. Specific attention is paid to others' affective experiences. This ethic of care supports the development of community and autonomy, and is emphasized in all three components of the LA Program.

The SPU LA Program defines learning as increasing participation in the discourse and practices of the community. The program instructor aims to relate the discourse and practices of LAs' own community to the broader education research community $[11,12]$. Learning is evident when LAs 'try on' research-based strategies, practices, and ways of thinking, as well as when LAs negotiate a shared language that connects the research literature to their teaching experiences.

This responsive, caring, participation-oriented context supported LAs in independently and collectively engaging with the mechanisms we propose. These mechanisms, in turn, fostered LAs' views of student ideas as sensible and productive.

\section{METHODOLOGY}

LAs in the SPU LA Program are required to submit weekly teaching reflections describing the impact of pedagogy or preparatory class discussions on their teaching practice. Over the course of two quarters during the 2012-2013 academic year, Robertson (the program instructor) noticed a shift in LAs' views toward student thinking. LAs began to employ language that ascribed intellectual value to student ideas by emphasizing the sensible and productive elements of student thinking.

For the analysis described in this paper, we culled reflections from LAs placed in introductory algebraand calculus-based physics during mid- to late-Winter 2013. Since we seek to explain the relationship between the shift and the mechanisms, the chosen reflections clearly and concisely illustrate connections between (a) LAs' interpretations of student thinking as intellectually valuable and (b) specific activities or events related to the LA pedagogy or prep courses. Thus, the quotes are not necessarily representative. However, they are not idiosyncratic: we found that the reflections written by seven of the nine LAs who were present during both Fall and Winter quarters indicated a view of student thinking as intellectually valuable.

\section{FOSTERING NOVICE TEACHER VIEWS OF STUDENT IDEAS AS SENSIBLE AND PRODUCTIVE}

For each mechanism described, we offer a general description as well as illustrative quotes from LA reflections that causally connect the mechanism to evidence of their intellectually valuing student ideas.

\section{LAs Articulate Teaching Values That Prioritize Student Reasoning}

Over the course of Winter Quarter (2013), LAs extensively discussed whether or not they think it is appropriate to leave an interaction with students whose answer is canonically incorrect. This question surfaced as LAs discussed Ball [8]. An LA asked, "Do we [LAs] think Ball is willing to let students walk away with wrong answers?" This question was prompted by Ball's explicit valuing of "Sean numbers," numbers (e.g., six) that "are both even and odd," since they contain an odd number of groups of two. The LAs decided that Ball would be willing to let students leave with a wrong answer since she did not try to change her class' consensus, but they were uncertain as to whether they were willing to do so in similar situations.

LAs extensively discussed under what circumstances and for what reasons it might be productive to leave students with a canonically incorrect answer. As a part of this conversation, they articulated stereotypes and nuances embedded in either stance: that it is either acceptable or unacceptable to leave students with the wrong answer. Emily's ${ }^{1}$ reflection illustrates how LAs eventually reconciled the opposing sides of this discussion. In this quote, she recognizes that student reasoning is central to both sides of the argument:

"I realized [that e] ach side had an exaggerated, inaccurate view of the other...The side that thought that it was okay to let students leave with the [wrong] answer viewed the side who said that it is not okay as forcing the right answer on students... and devaluing the students' ideas. The side that said that it was not okay viewed the other side as loosey-goosey hippies who would let students leave without fully formed ideas...These views of the other side are not true. Both evaluate critical thinking, and both have boundaries and circumstances, in which they are used."

\footnotetext{
${ }^{1}$ All names listed in this paper are pseudonyms, matched to the pseudonyms in Ref. 1.
} 
LAs explicitly connected their participation in this discussion to their perceptions of student ideas as intellectually valuable. For example, David reflects on video recordings of his teaching in the Fall versus his teaching in the Winter, saying:

"[M]y interaction [Fall] quarter was mainly me talking, asking leading questions and trying to direct student thinking in the way that I wanted it to go. How close minded of me! Those unique student ideas, even when incorrect, can provide so much insight, and usually offer a strong starting point on which to instruct that particular material...In our class discussions, we have talked about the...idea that student reasoning and understanding can be held in the highest priority, while simultaneously ensuring that the students walk away with correct answers..."

David explains that his teaching has shifted from explaining and asking leading questions to listening to and valuing student ideas as insightful, productive starting places for instruction. He connects this shift to the reconciliation of (a) his concern that students reach the correct answer and (b) the importance of student reasoning to his teaching.

LAs' teaching values did not necessarily shift to prioritize the student's reasoning over the canonical answer. However, the class debate seems to have significantly contributed to LAs' collective valuing of and attention to student reasoning.

\section{LAs Search for 'Kernels of Correctness'}

Near the beginning of the LA class discussion described in the previous section, the course instructor issued the following challenge to LAs: "I challenge you to make the assumption that there is a kernel of correctness in every answer that each student with whom you interact offers you. Before doing anything else, I challenge you to find that kernel and figure out why it is there or where it comes from." In issuing this "kernels of correctness" (KCs) challenge, the instructor requested that LAs 'try on' thinking of student ideas as sensible and as productive starting places for instruction, even when ideas are incorrect. LAs interpreted the phrase 'kernels of correctness' to mean: seeds of the canonical answer; productive resources on which to build instructionally; and sensible ideas based on students' experiences (regardless of their correctness).

The connection between intellectually valuing student ideas and the search for KCs is made explicit in Jess' reflection:

"...In one part of the tutorial it asks the students to compare tensions in [two] springs of different linear mass densities...When I was working with one student...[s]he said that Spring 1 had a greater tension because the wave speed was faster and that's what we saw in the first page. She also said that the spring just seemed tenser...This is true in the sense of how people feel tension...but this didn't necessarily fall in line with the physics definition of tension ...I had her make a free body diagram for the junction point. She then saw that there were only the 2 tension forces acting on it in the horizontal direction. Because the junction wasn't moving, she concluded that the acceleration was zero, thus the net force was zero [and] the tension between the 2 springs [was] the same...In this situation I really understood where she went wrong and just how it was easy to believe what she believed. I believe I learned how to analyze how students argue the [ir] point and find the kernel of truth in it and make it flourish from that."

Jess describes her student's (incorrect) idea as sensible: it makes sense to call Spring 1 tenser because greater tension often translates into greater pulse speed, and the spring has a 'tenser' appearance. Jess infers that the student is conflating tension and linear mass density, and her instructional intervention productively builds on the KCs she finds in student thinking (making them "flourish"). In general, this intentional search for KCs promoted LAs' careful consideration of what student reasoning has to offer, both in terms of its intrinsic value and its productivity.

\section{LAs Develop Curricular Knowledge}

SPU's introductory physics courses rely heavily on Tutorials in Introductory Physics [13]. These instructional materials embed particular strategies to build models for physics concepts and address common student difficulties $[14,15]$. During the weekly content preparation, LAs not only review the relevant Tutorial content; they also dissect the Tutorials pedagogically, seeking to understand the purpose of each particular section. For example, LAs agreed that Tutorials often address common student difficulties via an elicit, confront, resolve (ECR) sequence [16]. First, a common student difficulty is elicited. Next, the Tutorial shows the student that their initial idea contradicts a real-world example. Finally, the student resolves the inconsistencies between their original answer and the canonical answer.

One effect of the development of curricular knowledge was that LAs were empowered to partner with the curriculum to assess and respond to the learning needs of students. This represents a significant shift away from 'deployment' of a curriculum towards modifying or improvising instruction as necessary. Sarah describes the effect of her curricular knowledge as "freeing her up" to listen to student thinking: 
"Understanding the instructional strategies used by the tutorial has definitely [a]ffected my teaching because now I am less focused on the students knowing the exact answer because I feel it is more important for them to understand their thinking. I am also more comfortable in leaving the students to figure it out on their own, and I can just check their thinking at the end of each section."

Rather than solely assessing the correctness of students' answers to particular questions, Sarah focuses on the specifics of her students' ideas.

In a subsequent reflection, Sarah describes how she uses her knowledge of the curriculum's structure to facilitate an interaction:

"One student...thought that decreasing the distance between the two sources [in the Two Source Interference Tutorial] would increase the number of maximum interference lines. I decided to treat it like an ECR with her answer being the E...I pulled out one of the old pages of waves around a source point overlaid with a clear page so we could manipulate the source distances and see the lines. My C was explaining how the visual related to her question by putting my hand on the page to mimic the screen. Then she tested her prediction only to realize her prediction was wrong... [I]t was awesome because I [later] got to listen to how she resolved the previous observation as she answered this one correctly."

In this quote, Sarah describes an experiment she designed to test her student's idea. This demonstrates that her knowledge of the structure and goals of the curriculum gave her the freedom to modify instruction to suit the specific student.

\section{CONCLUSION}

This paper poses plausible mechanisms that fostered LAs' expanded perspective of student ideas as intellectually valuable. In our research with LAs in the SPU LA Program, we found that the debate as to whether or not it is acceptable to leave students with a wrong answer seemed to foreground the importance of student reasoning, which promoted attention to that reasoning. Also, the search for $\mathrm{KCs}$ embedded the assumption that student thinking was sensible and productive, and LAs who took this seriously embodied this mindset. Lastly, enhanced curricular knowledge enabled LAs to listen for the sense-making behind student answers. Although these mechanisms and the views they promote depend on the context of the SPU LA program, they illustrate what is possible when novice teachers engage in such development. As cases of novice teachers who intellectually value student ideas, students in the LA program show us what it looks like to act upon views of student thinking as sensible and productive, and how such views might develop.

\section{ACKNOWLEDGMENTS}

This material is based upon work supported by the National Science Foundation under Grant No. 0822342. We wish to thank C. Alvarado, A.R. Daane, L.S. DeWater, K.E. Gray, S.B. McKagan, R.E. Scherr, L.Seeley, and S. Vokos for their thoughtful feedback. We gratefully acknowledge the substantive contributions of LAs B. Clarke, A. Wing, A. Frazier, E. Maaske, L. Muñoz, J. Paige, K. Rininger, H. Sabo, C. Schmarr, F. Stewart, and S. Wenzinger.

\section{REFERENCES}

1. J. P. Smith III, A. A. diSessa, and J. Roschelle, J. Learn. Sci. 3 (2), 115-163 (1993).

2. T. P. Carpenter, E. Fennema, P. L. Peterson, C. Chiang, M. Loef, Am. Educ. Res. J. 26 (4), 499-531 (1989).

3. J. Pierson, "The Relationship Between Patterns of Classroom Discourse and Mathematics Learning," (University of Austin, Ph.D. dissertation, 2008).

4. V. Otero, S. Pollock, and N. Finkelstein, Am. J. Phys. 78 (11), 1218-1224 (2010).

5. J. E. Coffey, D. Hammer, D. M. Levin, and T. Grant, J. Res. Sci. Teach. 48 (10), 1109-1136 (2011).

6. E. Duckworth, "The having of wonderful ideas" and other essays on teaching and learning, New York: Teachers College Press, 2006.

7. D. Hammer, Cognition Instruct. 15 (4), 485-529 (1997).

8. D. L. Ball, Elem. School J. 93 (4), 373-397 (1993).

9. N. Noddings, Am. J. Educ. 96 (2), 215-230 (1988).

10. M. Mayeroff, On Caring, New York: Harper Perennial, 1971.

11. A. Sfard, Educ. Res. 27 (2), 4-13 (1998).

12. R. T. Putnam and H. Borko, Educ. Res. 29 (1), 4-15 (2000).

13. L. C. McDermott, P. S. Shaffer, and PEG at the UW, Tutorials in Introductory Physics, Prentice Hall, 2001.

14. L. C. McDermott, Am. J. Phys. 59 (4), 301-315 (1991).

15. P. R. L. Heron, "Empirical investigations of learning and teaching, part II: Developing research-based materials", in Proceedings of the International School of Physics "Enrico Fermi," Course CLVI, edited by E. F Redish and M. Vicentini (IOS Press, Amsterdam, 2004).

16. C. B. Cazden, Classroom Discourse: The Language of Teaching and Learning, Portsmouth: Heinemann, 2001. 